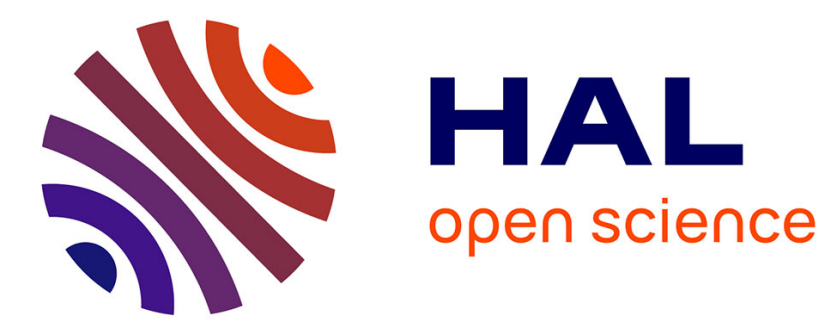

\title{
Sens et narrativité dans la société biographique
}

\author{
Christine Delory-Momberger
}

\section{To cite this version:}

Christine Delory-Momberger. Sens et narrativité dans la société biographique. Le sujet dans la Cité - Revue internationale de recherche biographique, 2012, Avoir droit dans la Cité. Vulnérabilités et pouvoir d'agir, 3, pp.167-181. hal-01158352

\section{HAL Id: hal-01158352 \\ https://hal.science/hal-01158352}

Submitted on 2 Jun 2015

HAL is a multi-disciplinary open access archive for the deposit and dissemination of scientific research documents, whether they are published or not. The documents may come from teaching and research institutions in France or abroad, or from public or private research centers.
L'archive ouverte pluridisciplinaire HAL, est destinée au dépôt et à la diffusion de documents scientifiques de niveau recherche, publiés ou non, émanant des établissements d'enseignement et de recherche français ou étrangers, des laboratoires publics ou privés.

\section{(이) $\$$}

Distributed under a Creative Commons Attribution - NonCommercial - NoDerivatives| 4.0 


\title{
sens et narrativité dans la société biographique
}

\author{
Christine Delory-Momberger
}

\section{Résumé}

Après avoir rappelé la dimension anthropologique du récit dans les associations humaines et pour les existences individuelles, la contribution examine les formes et les usages du récit de soi dans le contexte de la «société biographique » et interroge, à travers le modèle d'un «grand récit de l'individu », la relation entre activité narrative et production de sens.

\begin{abstract}
Meaning and narrativity in a biographical society

After recalling the anthropological dimension of narratives within human associations and for single lives, this paper examines the forms and use of self narratives in the context of " $a$ biographical society" and questions - throughout the model of a "great narrative of the individual"'- the relation between narrative activity and the production of meaning.
\end{abstract}

MOTS CLES : récit, narrativité, (auto)biographie, sens, société biographique.

KEYWORDS: narrative, narrativity, (auto)biography, meaning, biographical society.

Un des chapitres du livre de Wilhelm Schapp ${ }^{1}$, Empêtrés dans des histoires (1992 [1953]), porte ce titre remarquable : «Aperçu sur la question de savoir s'il peut y avoir quelque chose

\footnotetext{
${ }^{1}$ Wilhelm Schapp (1884-1969), élève de Dilthey et de Husserl, philosophe et juriste de métier, fut membre du Cercle des phénoménologues de Göttingen. Auteur de la première Phénoménologie de la perception, il mène des recherches pour une fondation phénoménologique de la philosophie du droit (1930-1932) et élabore, à partir de 1950, une phénoménologie de la narrativité qui donne naissance à son ouvrage In Geschichten verstrickt. Zum Sein von Mensch und Ding (1953).
} 
en dehors des histoires ». Dans le contexte de l'œuvre de Schapp, cette question est largement rhétorique et appelle à l'évidence une réponse négative : non, il n'y a rien en dehors des histoires, pour Schapp tout commence et, pourrait-on dire, tout finit avec les histoires. On sait que personne n'aura poussé plus loin, et d'une manière aussi radicale, l'entreprise d'établir une «anthropologie narrative ». Il étend même cette emprise - cet empire - des histoires à l'ensemble des « réalités » dont s'occupent les sciences physiques et les sciences naturelles : la matière et la matérialité, dit-il, surgissent seulement dans des histoires, comme si les histoires engendraient en quelque sorte le réel. Le monde physique, biologique, humain ne prend forme et ne prend sens qu'avec les récits que les hommes, dans leur condition d' "êtres empêtrés dans des histoires », racontent à son propos.

W. Schapp établissait ainsi, en termes à la fois anthropologiques et phénoménologiques, le rapport de sens que les hommes entretiennent avec le monde à travers les récits qu'ils en font, rapport illustré originairement, dans toutes les cultures du monde, par les mythologies, et en particulier par les mythes étiologiques qui racontent l'origine des choses et des êtres. A l'ère du développement des sciences humaines, c'est sur ce même principe de l'intelligibilité narrative que Wilhelm Dilthey (1992 [1883]; 1988 [1910]) avait fondé sa réflexion épistémologique, en faisant de la réflexivité et du récit autobiographiques le fondement de la compréhension déployée par les «sciences de l'esprit». Et c'est encore sur ce mode de compréhension par les histoires que s'appuient certaines approches culturelles et pédagogiques, comme celles de Jerome Bruner qui recourt au récit en tant que mode de pensée et d'intelligibilité du monde et de soi dans le monde : à travers le récit on apprend à analyser la réalité, à organiser et à comprendre le monde dans lequel on vit, le monde naturel autant que le monde social ${ }^{2}$. Tant le monde de significations que les êtres humains, pris en tant qu'espèce ou en tant qu'individus, donnent à eux-mêmes, à leur existence, aux formes de leurs associations, à leurs manières de vivre ensemble, à l'univers qui les entoure, - tant ce monde de significations trouve sa forme, et plus que sa forme, son lieu, dans les récits et les histoires que les hommes se racontent.

Comme son titre le laisse entendre, le propos de cette contribution est d'examiner quelles sont les formes de récit auxquelles est lié le sens de la vie collective comme celui de la vie individuelle dans le cadre de nos sociétés et de nos existences contemporaines. Mais avant d'en venir à cette contextualisation socio-historique, il ne paraît pas inutile de rappeler, d'une

\footnotetext{
2 «L'aptitude à construire et à comprendre des récits est essentielle dans la construction de notre vie et d'un « lieu » qui nous soit personnel dans le monde que nous allons affronter [...]. Ce n'est que par le mode narratif que l'on peut construire cette identité et trouver une place dans sa propre culture. » J. Bruner, 1996, p. 59 et p. 61.
} 
manière plus atemporelle, la relation entre l'activité narrative et la production de sens, entre le fait de recourir au discours du récit et le fait de donner du sens à ce dont il est question dans le récit, à savoir des existences et des expériences humaines, des actions et des vouloirs humains.

\section{Comment le récit donne forme à l'existence}

Quels sont les ressorts et les caractéristiques formelles qui donnent au récit, en tant qu'activité langagière et que forme spécifique de discours, la faculté de produire du sens, ou plus exactement des formes qui font sens ? Ce qui est en jeu à travers cette question, c'est la manière dont les êtres humains créent de la signification, dont ils donnent forme à leur expérience et dont ils comprennent le monde et leur propre existence.

Le rapport constitutif du récit à la vie humaine est ancré dans la dimension du temps. L'être humain fait l'expérience de sa vie et de lui-même dans le temps. Il éprouve son existence dans le sentiment d'une unité et d'une identité maintenues à travers le temps. Les êtres humains n'ont cependant pas un rapport direct, transparent à leur vécu et au déroulement de leur vie ; ce rapport est médiatisé par le langage et par ses formes symboliques. Pour figurer le déploiement temporel de leur vie, les hommes recourent à des mots et à des images qui ont pour point commun de désigner un espace à parcourir dans le temps : ligne, fil, chemin, parcours, cercle, cycle, carrière de la vie. L'homme écrit dans l'espace la figure temporelle de son existence. Les images dont il se sert pour en évoquer le cours (à commencer par celle-ci) lui sont cependant tellement familières qu'elles ont perdu leur dimension symbolique ou analogique et qu'elles en viennent à faire oublier que ce n'est que par métaphore, selon les voies d'une écriture, que de telles figures peuvent être prêtées à son existence.

C'est sur le fond de cette temporalité de l'expérience humaine médiatisée par le langage que le récit vient trouver son inscription et son statut anthropologiques. C'est toute l'entreprise théorique de Paul Ricoeur dans Temps et récit de montrer quelles sont les corrélations entre l'activité de raconter une histoire et le caractère temporel de l'expérience humaine : «Le temps devient temps humain, écrit-il, dans la mesure où il est articulé de manière narrative ; en retour le récit est significatif dans la mesure où il dessine les traits de l'expérience temporelle»(Ricoeur, 1983, p. 17). L'espace-temps selon lequel nous nous figurons les bornes de notre existence est en effet celui sur lequel naissent nos histoires, c'est- 
à-dire les constructions narratives selon lesquelles nous appréhendons notre vie. Dès que nous voulons nous saisir de notre vie, nous la racontons. Nous n'avons pas d'autre moyen pour accéder à notre vie que de percevoir ce que nous vivons à travers l'écriture d'une histoire (ou d'une multiplicité d'histoires) : en quelque sorte nous ne vivons notre vie qu'autant que nous l'écrivons dans le langage des histoires. Pour citer encore Paul Ricoeur : «Il n’y a pas d'expérience humaine, observe-t-il, qui ne soit déjà médiatisée par des systèmes symboliques et, parmi eux, par des récits [...] Nous n'avons pas d'accès aux drames temporels de l'existence en dehors des histoires racontées à leur sujet par d'autres ou par nous-mêmes. » (1983, p. 113)

De quelles caractéristiques internes le discours narratif tient-il une telle efficience signifiante ? On se contentera ici de quelques brefs éléments de réponse. Le récit introduit dans la pure succession temporelle une organisation - une syntaxe - bien particulière fondée sur la causalité et la finalité : dans le récit, non seulement les situations, les événements se passent les uns après les autres, mais ils sont articulés selon des relations de cause à effet réalisant la formulation aristotélicienne du post hoc propter hoc, qui tire un rapport de causalité d'une relation de succession - et ils sont organisés pour aller vers une fin : ils forment ainsi une intrigue qui a un début, un développement, un terme. Le récit transforme les événements, les actions et les personnes du vécu en épisodes, en intrigues et en personnages; il ordonne les événements dans le temps et construit entre eux des relations de cause, de conséquence, de but, il donne ainsi une place et un sens à l'occasionnel, au fortuit, à l'hétérogène. Par le récit, les hommes se font les propres personnages de leur vie et donnent à celle-ci une histoire.

Cette opération de configuration, que Paul Ricoeur a décrite sous le terme de mise en intrigue $^{3}$, s'accomplit dans et par le langage. C'est le récit, en tant que genre du discours, qui en est, non seulement le moyen, mais le lieu : la vie a lieu dans le récit, et elle a lieu en tant qu'histoire. Ce qui donne forme au vécu et à l'expérience des hommes, ce sont les récits qu'ils en font. Le narratif n'est donc pas seulement le système symbolique dans lequel les hommes trouvent à exprimer le sentiment de leur existence : le narratif est le lieu où l'existence humaine prend forme, où elle s'élabore et s'expérimente sous la forme d'une histoire.

Le récit constitue ainsi une capacité fondamentale de l'espèce humaine et joue un rôle essentiel dans la constitution de l'expérience. Pour citer des références déjà anciennes mais

\footnotetext{
${ }^{3}$ La mise en intrigue transforme une diversité d'événements ou d'incidents successifs en une histoire organisée et prise comme un tout : «Une histoire doit être plus qu'une énumération d'événements dans un ordre sériel, elle doit les organiser dans une totalité intelligible [...]. La mise en intrigue est l'opération qui tire d'une simple succession une configuration.» P. Ricoeur, 1983, p. 102.
} 
toujours valides, Bergson (1967) voit dans ce qu'il nomme la fonction fabulatrice « une faculté bien définie de l'esprit, celle de créer des personnages dont nous nous racontons à nous-mêmes l'histoire » (p. 205), et reconnaît dans cette faculté « une fonction indispensable à l'existence des individus comme à celle des sociétés » (p. 207). Et le psychologue Pierre Janet (1935; 1936) montre l'importance, pour le développement de l'enfant comme pour la gestion adulte des relations interindividuelles et sociales, des conduites de récit qui permettent de se mettre à la place d'autrui, de se représenter ses sentiments et ses intentions et de comprendre ses actions dans le cadre d'une histoire. La re-présentation narrative offre en effet le cadre concret d'une mise en scène de l'action et de l'intentionnalité humaines, d'une ontologie en acte de l'humain. Certains travaux contemporains des spécialistes des neurosciences font de la compétence narrative une donnée biologique, une composante de l'équipement cognitif de l'être humain, en postulant dans le cerveau l'existence d'un module « interpréteur », dont la fonction est de donner un sens aux événements en les intégrant dans des récits où ils sont liés par des chaînes causales et intentionnelles (Gazzaniga, 1994 ; 2008), ou en faisant l'hypothèse d'une structure narrative de la conscience (Dennett, 1995).

Cette capacité anthropologique selon laquelle les hommes perçoivent leur vie et ordonne leur expérience dans les termes d'une raison narrative doit être ici entendue comme une attitude première et spécifique du vécu humain : avant même de laisser de notre vie une quelconque trace écrite, avant tout discours, oral ou écrit, tenu sur nous-mêmes, nous configurons mentalement notre vie dans la syntaxe du récit. La perception et l'intelligence de notre vécu passent par des représentations qui prêtent une figure narrative aux événements et aux situations de notre existence. Pour introduire des termes que nous utilisons en recherche biographique, nous n'arrêtons pas de nous biographier, c'est-à-dire d'inscrire notre expérience dans des schémas temporels orientés qui organisent mentalement nos gestes, nos comportements, nos actions, selon une logique de configuration narrative. Cette activité de biographisation pourrait être définie comme une dimension du penser et de l'agir humain qui, sous la forme d'une herméneutique pratique, permet aux individus, dans les conditions de leurs inscriptions socio-historiques, d'intégrer, de structurer, d'interpréter les situations et les événements de leur vécu (Delory-Momberger, 2005).

\section{Les récits font les associations humaines}


Ce qui vaut pour l'existence individuelle vaut également pour toutes les formes de récit par lesquelles les êtres humains racontent leur «histoire» collective, leur «cité », leur «société », leur «culture », etc. Dans le pouvoir d'élaboration et de construction du récit, il faut reconnaître une forme particulière de pensée qui a une dimension anthropologique. Si penser, c'est associer, articuler, relier, ordonner, alors le langage du récit représente une forme spécifique de la pensée, qui consiste à articuler des actions dans le temps selon des enchaînements de causalité et de finalité. Cette forme de pensée semble répondre à un mécanisme universel, à une donnée que l'on retrouve dans toutes les cultures et à toutes les époques, qui accompagne l'évolution de l'espèce (phylogenèse) comme elle accompagne l'évolution de l'individu (ontogenèse) : l'homme est un «être racontant» (homo narrans) l'espèce humaine une « espèce racontante », la seule qui se raconte elle-même et dont chacun des membres puisse se reconnaître une « histoire $»^{4}$. «Le "pouvoir raconter", écrit Ricoeur (2005), occupe une place éminente parmi les capacités humaines dans la mesure où les événements de toute origine ne deviennent lisibles et intelligibles que racontés dans des histoires. »

Les récits en tant que mode d'intelligibilité de l'expérience humaine et du monde sont au cœur des cultures et des sociétés. Ce sont les récits qui procurent des modèles de compréhension de l'action collective et individuelle. Il faut essayer de mesurer les enjeux d'une telle présence du récit. Il n'y a pas de collectivité, de communauté humaine sans récit, il n’y a pas d'espace social sans récit, il n'y a pas de «territoire » sans récit. Toutes les formes d'association humaine s'édifient sur des récits : récits de fondation ou d'origine, mythes, légendes, histoires nationales, etc. Etre en société, vivre ensemble, c'est partager les mêmes récits. Et cela concerne toutes les échelles d'association et d'établissement humains : du niveau le plus large, celui des aires de civilisation (l'Occident et ses «grands récits » : récit chrétien de la chute et du salut, récit « grec » du logos et de la raison, récit du progrès, récits d'émancipation, récit «communiste» de l'avènement de la société sans classe) au niveau intermédiaire du pays et de la nation («histoires nationales », «grands hommes », amis et surtout ennemis héréditaires), jusqu'au niveau le plus étroit et intime du couple et de la famille (récits des «premières fois », « histoires familiales », « secrets de famille »).

Mais cela concerne aussi les groupes sociaux (récits de la classe ouvrière, récits du peuple en lutte), les catégories socioprofessionnelles (histoires de métier, « récits de pratiques»), les groupes de convivialité ou de sociabilité. Et cela concerne également les

\footnotetext{
${ }^{4}$ Selon le linguiste Bernard Victorri (2002), la fonction narrative est à l'origine de l'émergence du langage et du développement par l'espèce Homo sapiens des formes d'organisation qui régissent les sociétés humaines.
} 
espaces humains, ce que les géographes des sociétés appellent l'«habiter»: il n'y pas de «lieu habité », il n’y a pas de «territoire » sans récits partagés par ceux qui y vivent et les habitent. Quelle que soit la forme de reliance que les individus ont entre eux, cette reliance est toujours, non seulement médiatisée par des récits, mais aussi fondée sur des histoires qui racontent l'origine, qui font partager les mêmes expériences, les mêmes situations, qui engagent des motivations communes et des intérêts communs, qui indiquent des objectifs, donnent des orientation, tracent les voies d'un avenir.

Ces récits ne vont pas tous forcément dans le même sens, ils peuvent présenter des « histoires », et donc des interprétations différentes ou opposées d'une même réalité. Entre ces récits collectifs, il peut y avoir concurrence ou contradiction entre des versions ou des visions différentes de la réalité sociale et politique (récit de «la droite » et récit de «la gauche», scandale d'un président de droite qui «vole» au récit de la gauche quelques-unes de ses grandes figures, Blum, Jaurès ou Guy Moquet) ; concurrence ou contradiction d'échelle et de points de vue entre les lieux d'où l'on tient le récit (par exemple, le récit étatique des politiques sociales versus le récit des professionnels du social sur le terrain par rapport au « chômage », à l' « exclusion », à l' « insertion » versus les récits tenus par les personnes qui se trouvent elles-mêmes dans ces situations et qui sont «assignées »dans des figures de chômeurs ou d'exclus).

Les contradictions entre les récits collectifs reflètent et produisent les contradictions sociétales et les conflits politiques. Elles se répercutent dans les existences individuelles et dans les représentations et les constructions biographiques. S'il n'y a pas de «société humaine » sans récit, il n'y pas non plus de sentiment individuel d'appartenance à un groupe ou à une collectivité sans partage de récit, il n'y a pas d'identité individuelle, personnelle et/ou professionnelle sans adhésion ou au moins relation aux récits collectifs propres aux groupes auxquels on appartient. Le récit est un facteur de cohésion sociale comme il est un moteur de cohérence et d'identité individuelle, mais l'identité narrative individuelle se construit toujours dans une relation aux récits collectifs (récits officiels ou institutionnels, récit des autres). Le récit individuel est le lieu d'une tension, d'une négociation, d'une transaction entre un ensemble de récits collectifs, ceux des groupes et collectivités sociales d'inscription ou d'appartenance, et une trajectoire individuelle singulière, un sentiment unique de soi-même et de son existence.

C'est dire que, sur ce fond anthropologique que nous venons d'évoquer, il convient assurément de réintroduire une perspective historique et culturelle. Comme tous les systèmes symboliques, les récits sont soumis aux variations sociohistoriques : les modèles et les formes 
de récit auxquelles les individus empruntent pour biographier leur vie ne leur appartiennent pas en propre, ils ne peuvent pas en décider entièrement par eux-mêmes, ce sont des formes collectives qui reflètent et conditionnent à la fois les rapports qu'à telle époque et au sein de telle culture les individus entretiennent avec la collectivité et avec eux-mêmes ${ }^{5}$. Les modèles narratifs servent ainsi de patrons biographiques aux constructions individuelles. Ils procurent aux individus des schémas et des modalités de relation à eux-mêmes et d'insertion au sein de la collectivité, et ils accompagnent les évolutions sociétales dans ce domaine.

\section{Fin ou métamorphoses des grands récits dans la modernité avancée ?}

Ce propos conduit à interroger les formes que prennent aujourd'hui les récits collectifs et les constructions narratives (biographiques) individuelles. Depuis que François Lyotard (1979) en a théorisé la fin il a plus de trente ans, l'habitude s'est prise d'opposer aux « grands récits » de la modernité le «petit récit» d'un individu postmoderne privé des références et des ressources idéologiques, politiques, morales, spirituelles qu'offraient précisément les grands récits, et réduit, abandonné, à la «petite affaire » de sa vie dans une société managériale et néolibérale n'offrant plus d'autres « valeurs » que celles de la consommation et de la réussite matérielles. Pour n'en donner qu'un exemple récent, Dany-Robert Dufour (2011) voit dans la société du marché érigé en maître tout-puissant («le divin Marché ») le triomphe du récit du self love sur le récit du dépassement de soi, triomphe qui marque l'issue d'un long combat entamé depuis plus de deux siècles entre les Lumières allemandes et les Lumières anglaises, entre une préoccupation de transcendance et l'intérêt individuel, entre Kant et Adam Smith.

Ce que l'on souhaiterait montrer ici, c'est que ce «petit récit», dont on fait volontiers grief à l'individu de la postmodernité - ou du moins à la forme de société qui le produit -, relève bien lui aussi de formes de grand récit, tout comme le «récit de confession » de tel clerc du Moyen Âge relevait du grand récit chrétien du Salut ou le « récit de lutte» d'un ouvrier des années 1950 relevait d'un grand récit communiste de l'avènement de la société sans classe. Et peut-être sera-t-on amené à contester ou au moins à nuancer cette lecture de la fin des grands récits engagée à la fin des années 1970 et qui suscite depuis un très large consensus. Si en effet la thèse de la fin des grands récits est en partie vérifiée - au moins dans le monde occidental - pour les formes historiques de récit collectif auxquelles les individus, en fonction de leurs appartenances sociales et de leurs attaches idéologiques, pouvaient

\footnotetext{
${ }^{5}$ Pour une histoire de ces formes collectives du récit de soi, cf. C. Delory-Momberger, 2004.
} 
rapporter leur existence, on peut cependant s'interroger sur l'émergence et le développement dans la modernité avancée de nouvelles matrices narratives, au caractère historique et idéologique moins directement lisible, venues d'autres sources et d'autres espaces et s'exerçant selon des modes opératoires sensiblement différents.

Les formes nouvelles de métadiscours que suscite la modernité avancée ont en effet pour origine d'autres territoires que ceux de la croyance religieuse et de l'idéologie politique, fournisseurs traditionnels de mythes et de grands récits. C'est en partie dans le champ des sciences humaines et sociales que se construisent aujourd'hui les représentations que se font d'eux-mêmes les sociétés et les individus. Les sociétés de la modernité avancée sont devenues des sociétés réflexives qui produisent à leur propre endroit un savoir et un discours qui ont des effets régulateurs sur les conduites collectives et individuelles. La réflexivité entendue comme la capacité propre aux acteurs humains de «comprendre ce qu'ils font pendant qu'ils le font » (Giddens, 1994, p. 33) est une des caractéristiques majeures de la vie sociale moderne, où elle se traduit par «l'examen et la révision constante des pratiques sociales à la lumière des informations nouvelles concernant ces pratiques, ce qui altère aussi constitutivement leur caractère »(Ibidem, p. 45). Les savoirs et les discours des diverses sciences humaines, en particulier ceux de l'histoire, de l'anthropologie et de la sociologie, qui ont pour ambition de décrire et d'analyser les modes du vivre-ensemble contemporain et leur évolution, sont repris dans le cadre sociétal et réinterprétés sur un mode normatif et prescriptif. Les sciences sociales participent ainsi au premier chef à la construction des représentations de la société et à l'élaboration des comportements sociaux. Par une véritable intrusion du discours expert dans la vie ordinaire se répandent des descriptions du «monde humain » qui orientent et structurent les conduites individuelles. Véhiculés tant par les médias que par les institutions politiques, sociales et économiques, ces métadiscours contribuent à construire l'imaginaire sociétal de l'individu contemporain, à la fois inscrit dans un espace-temps physique et mental ouvert sur la globalité du monde et disposant d'une capacité de réflexivité sur lui-même, d'autonomie de son action et de production de son histoire. «Ruse de la raison » ou plutôt ruse de la société, les formes de grand récit qui prennent le pas dans nos sociétés contemporaines coïncident avec l'injonction narrative de la «réalisation individuelle ». Autrement dit, dans le modèle de récit qui informe aujourd'hui les modes de conduite et d'existence, « l'individu » en tant que tel - souvent anobli en « acteur » ou en «sujet»-s'est substitué aux figures et aux valeurs qui étaient celles des grands récits historiques de la modernité. Mais tout comme ses prédécesseurs, ce modèle du « grand récit de l'individu » est 
le fruit d'une production socio-historique et idéologique, dont il faut chercher le creuset dans la société néolibérale.

\section{Discours de l'autoréalisation et société néolibérale}

On peut tenter de donner quelques éléments pour situer l'émergence du discours de l'autoréalisation et pour comprendre la place qu'il a prise dans la conduite de la vie collective et individuelle. Le discours de l'autoréalisation et l'injonction biographique qui en résulte sont liés à ce que l'on pourrait décrire comme un « changement de régime » dans le rapport de l'individu et du social. Cette mutation qui affecte les sociétés modernes a fait l'objet d'analyses qui sont maintenant largement répandues (Giddens, Beck, Bauman, Ehrenberg), dont il ressort que l'individu est devenu l'institution centrale de la société et qu'il lui revient, pour reprendre la formule d'Ulrich Beck, d'être «l'unité de reproduction de la sphère sociale » (2003, p. 280). A la socialisation conçue comme intégration des normes sociales et accomplissement des rôles sociaux succèdent des formes nouvelles de sociétisation, faisant une part de plus en plus grande à l'expérience individuelle et conduisant les individus à se placer eux-mêmes au centre de leur propre plan d'existence ${ }^{6}$. J'ai pour ma part désigné sous le terme de condition biographique cette inflexion du rapport historique entre l'individu et le social, dans laquelle les individus imputent à une responsabilité personnelle les conséquences sur leurs existences de dépendances et de contraintes extérieures (institutionnelles, sociales, économiques) et sont enjoints à trouver en eux-mêmes les ressorts de leur conduite et de leur action (Delory-Momberger, 2009).

Un tel renversement dans le rapport de l'individu et du social n'est pas exempt d'effets contradictoires et d'injonctions paradoxales. D'un côté, l'individualisme sociétal s'accompagne d'un accroissement de la «conscience de soi » et de la représentation partagée selon laquelle les individus sont capables, par leur activité réflexive et interprétative, d'agir sur eux-mêmes et sur leur vie, de donner une forme personnelle à leurs inscriptions sociales, de choisir sinon leur destin, du moins leur existence. Ce rapport réflexif se traduit par une aspiration à la réalisation personnelle et par un regard autre porté sur le déroulement et sur le sens d'une existence ouverte à de multiples possibles. Mais d'un autre côté, cet individualisme réflexif est prompt à se renverser en individualisme normatif, dès lors que ses

\footnotetext{
6 « La socialisation, peut écrire Michel Wievorka, [...] est aujourd'hui avant tout le processus dans lequel [un individu] acquiert et développe sa capacité à maîtriser son expérience, à être sujet de son existence. ». Wieviorka, 2008, p. 319)
} 
motifs (responsabilité, autonomie, réalisation de soi) sont récupérés, en particulier par le monde de l'entreprise libérale, à des fins de rentabilité économique et de «gestion des ressources humaines ». Il se mue alors en idéologie de l'excellence et de la performance et en instrument de conformation : ce qui était aspirations individuelles se transforme en schémas et en modèles institués que les individus rencontrent désormais comme des exigences venues de l'extérieur auxquels ils se doivent de conformer leur parcours, leurs comportements personnels et professionnels.

Une des formes les plus radicales que prend ce modèle de l'autoréalisation est celle de l'homo aconomicus de la société néolibérale, tel par exemple que Pierre Dardot et Christian Laval en dressent le portrait dans leur ouvrage La Nouvelle raison moderne (2009). Héritière de la doctrine de la «main invisible », la psychosociologie néolibérale fait de l'individu un analogon de l'entreprise de libre marché : les individus n'ont d'autres visées que la satisfaction d'intérêts «égoïstes » et mettent à la poursuite de ces intérêts les ressources d'une raison calculatrice ${ }^{7}$. La société-entreprise porte en son sein la figure d'un sujet « entrepreneur de lui-même » qui met toute son énergie à «investir ses ressources », à «gérer son portefeuille d'activités », à «faire fructifier son capital humain ». Pierre Dardot et Christian Laval s'attachent à montrer que ces assimilations de l'individu à l'entreprise, loin de constituer de simples métaphores, relèvent d'un nouveau «discours de l'homme » et définissent une nouvelle norme subjective : «'Entreprise', écrivent-ils, est le nom que l'on doit donner au gouvernement de soi à l'âge néolibéral. » (p. 409) La norme de performance et de réalisation individuelle du sujet néolibéral s'inscrit dans un ordre mondial de la compétition économique, où elle trouve en dernier ressort sa justification : le microcosme de l'«entreprise de soi », réplique du monde de l'entreprise, répond au macrocosme du marché planétaire. Les métadiscours de la globalisation économique et de l'homo æeconomicus renvoient ainsi l'un à l'autre pour produire la figure d'un sujet rationnel et calculateur œuvrant à la réussite entrepreneuriale de sa propre existence. Cette injonction biographique ne manque pas de se parer de valeurs «humanistes », qu'il s'agisse de l'«autonomie », de la « réflexivité », de la « responsabilité », de la « réalisation de soi », etc.

\section{Centralité sociale du récit de soi et injonction du sens}

\footnotetext{
7 «Dans le néolibéralisme avancé, l'individu n'est autre que le nom même du lien social régulé par la loi du profit et de l'intérêt. » M. Benasayag, 2004, p. 28.
} 
Ce qu'il faut également retenir de ce changement de régime dans le rapport de l'individu et du social, ce sont les transformations qui affectent le statut et les pratiques du récit de soi. Dans cette nouvelle configuration sociétale, le récit de soi n'est plus seulement le lieu d'un usage «privé » et d'un enjeu d'expression et de construction personnelles : il fait l'objet d'un véritable impératif social et il se charge d'enjeux nouveaux en relation tant avec sa fonction d'élaboration de l'expérience individuelle et collective qu'avec ses usages dans l'espace public.

Dans ce contexte, le récit de soi acquiert une centralité sociale qu'il n'avait sans doute jamais eue jusqu'ici : il n'est plus seulement une forme de l'expression personnelle, un lieu d'exploration de l'intimité et de l'intériorité, un support de connaissance ou de découverte de soi-même - toutes pratiques qui en situaient l'usage dans l'espace du privé ; il passe résolument dans la sphère publique, il devient un instrument essentiel de la médiation, de la reconnaissance, de la contrepartie sociale (voir les formes de contractualisation biographique à l'œuvre dans les politiques sociales : donne-moi ton récit et je te donnerai une allocation, un stage $^{8}$ ); mais il aussi le lieu où s'élabore cette « reproduction de la sphère sociale »dont l'individu est devenu lui-même l'unité, pour reprendre la formulation de Beck.

Le récit de soi porte ainsi les marques forcément ambivalentes des usages dont il est l'objet, des fonctions qu'il est amené à investir : tantôt support de construction personnelle, tantôt objet public formaté et réifié ; tantôt lieu d'élaboration de l'expérience singulière, tantôt lieu de conformation; tantôt acte de résistance, tantôt acte d'allégeance ; tantôt récit qui fait advenir un «sujet», tantôt récit qui assujettit. Le récit de soi semble toujours pris dans une tension entre assujettissement et subjectivation, entre bio-politique comme gouvernement des corps et de la vie, comme pouvoir de «conduire les conduites » (Foucault, 2001, p. 1056), et auto-bio-graphie comme effort du sujet de se constituer lui-même et de donner une forme à son expérience.

Mais au-delà de ces polarités, au-delà également des nouvelles inégalités liées à la capacité individuelle et collective de faire récit de sa vie et à celle d'être entendu, ce qu'il convient de retenir ici, c'est cette centralité sociale ou sociétale du récit biographique, lorsque - pour le dire d'une formule - l'homo socius est constitué en homo narrans. Dans une société de «rapports sociaux de production de soi »(Franssen, 2006, p. 75), où les individus sont enjoints de produire le monde social à partir de leurs expériences singulières, les constructions narratives selon lesquelles ils travaillent à donner forme à leurs expériences et participent ce

\footnotetext{
8 Sur ces questions, voir notamment Isabelle Astier, 2007.
} 
faisant à la production de l'espace social se chargent d'une signification nouvelle et acquièrent une dimension sociétale.

On peut penser que cette injonction adressée à chacun et intériorisée par chacun de «faire histoire de sa vie », que cette mutation du récit de soi en impératif à la fois moral et social marquent un tournant dont nous n'avons pas encore complètement pris la mesure. Car ce qui s'impose avec cet impératif du récit, c'est aussi l'injonction du sens, l'injonction d'avoir à trouver et à formuler le sens de sa propre existence dans une prise de forme de son histoire et de son expérience. Il s'agit là d'un fait inédit dans l'histoire des sociétés : jamais aucune société n'a demandé à ce point à l'ensemble de ses membres de "produire » (au sens à la fois de fabriquer et de rendre visible) individuellement le sens de leur existence. La charge de sens qui pèse aujourd'hui sur les individus est considérable: on demande à l'écolier, à l'étudiant, à l'ouvrier, à l'employé, etc. de faire état du sens qu'il donne à sa situation, à son travail, à son parcours, à son avenir ; la même exigence réflexive est adressée à «l'homme » et à «la femme », au père, à la mère, aux grands-parents, etc., et encore à l'électeur, au parent d'élève, au consommateur, au citoyen.

La même charge de sens pèse sur la collectivité. Les sociétés réflexives produisent un savoir régulateur sur elles-mêmes mais aussi des questionnements sur leur propre sens et sur la dimension du sens dans leur fonctionnement. Une telle mise en réflexivité ne semble pas avoir été possible dans des formes de sociétés antérieures, qu'il s'agisse des sociétés premières, des sociétés antiques ou même des sociétés de la première modernité. Les Romains se sont beaucoup interrogés sur le déclin de leur empire et l'ont mis au compte de diverses raisons morales et politiques. Mais ils ne pouvaient se mettre dans la posture d'abstraction réflexive qui les aurait amenés à se demander si l'empire romain avait besoin de sens pour se perpétuer : la question n'aurait pas eu de sens ou plutôt le sentiment d'évidence et le jugement de tautologie qu'elle aurait appelés l'auraient privée de toute pertinence et l'auraient rendue proprement inconcevable. Et sans doute n'aurait-elle pas eu beaucoup plus de sens dans nos propres sociétés européennes du XVII ${ }^{\mathrm{e}}$ ou du XVIII ${ }^{\mathrm{e}}$ siècle. Le questionnement sur le sens et la requête de production de sens que les sociétés de la modernité avancée s'adressent à ellesmêmes est symptomatique de la même révolution dont témoignent l'injonction biographique et la charge de sens adressés aux individus.

\section{Conclusion}


Alors que le sujet moderne trouvait dans les «grands récits » des références de sens et des modèles de conduite en fonction de ses inscriptions, de ses affiliations, de ses appartenances, l'individu de la modernité avancée est renvoyé à lui-même et à son propre récit pour produire le sens de son expérience. Il lui revient de trouver par lui-même son lieu, en reliant et en faisant signifier les espaces sociaux auxquels il participe. Or, cette façon de faire sens et de faire lieu en lui-même, il ne peut la trouver que dans un rapport réflexif à sa propre existence. La construction biographique de l'expérience - c'est-à-dire la capacité à intégrer dans le continuum d'une histoire les événements et les situations de l'existence - apparaît ainsi comme la médiation nécessaire d'un monde dont les repères et les métadiscours sont brouillés ou décrédibilisés, d'un monde qui ne peut être relié que dans la réflexivité et l'historicisation de l'expérience qu'en font les individus.

C'est dans ce sens que l'on peut parler aujourd'hui d'un «grand récit » qui ne serait plus un « récit du monde » ou un «récit de la société », mais un « récit de l'individu » en tant qu'il fait société et en tant qu'il fait monde, en tant qu'il lui incombe de reproduire la sphère sociale et de médier le monde. C'est sans doute à cette redéfinition du sujet sociétal que le récit de formation doit sa pérennité jusque dans nos représentations et constructions contemporaines : il y retrouve même une exemplarité et une vigueur renouvelées, puisque la figure qu'il met en scène d'un individu qui forme le monde en se formant lui-même rejoint en un sens l'intuition originelle des Lumières et de la Bildung romantique. Ce qui l'en différencie cependant de manière décisive, c'est que ce récit vaut pour lui-même et en lui-même, qu'il n'est plus soutenu par les grands discours de légitimation (la raison, le progrès, l'émancipation) qui l'ont longtemps accompagné, en lui fournissant valeurs et justifications. Dans ce nouveau grand récit, l'individu est enjoint de tirer de lui-même les ressorts de son action et les principes de sa conduite, de construire les motifs et les valeurs qui donnent sens à son action, de nouer les «associations » qui le lient aux autres et qui lui donnent une place dans le tissu des collectivités. Récit tout aussi «mythique », mais aux effets tout aussi réels que ceux qui l'ont précédé, ce «grand récit de l'existence individuelle » est une construction socio-historique, une des formes selon lesquelles la société se représente elle-même et assigne à ses membres leur tâche et la manière de la remplir. Relayant les grands récits historiques de la modernité, c'est cette mise en intrigue d'une individualité puisant en elle-même ses ressources et ses valeurs qui structure et oriente aujourd'hui les modes de représentation et de construction des existences, c'est dans cette figure d'un sujet capable de faire monde par luimême que prend effet la société biographique. 


\section{Références bibliographiques}

Astier, I. (2007). Les nouvelles règles du social. Paris : Presses Universitaires de France.

Beck, U. (2003). La Société du risque. Sur la voie d'une autre modernité. Paris: Champs Flammarion.

Benasayag, M. (2004). Le Mythe de l'individu. Paris : La Découverte/Poche.

Bergson, H. (1967 [1932]). Les Deux sources de la morale et de la religion. Paris : Presses Universitaires de France.

Bruner, J. (1996). L'éducation, entrée dans la culture. Les problèmes de l'école à la lumière de la psychologie culturelle. Paris : Retz.

Dardot, P. \& Laval, C (2009). La Nouvelle raison moderne. Essai sur la société néolibérale. Paris : La Découverte.

Delory-Momberger, C. (2004). Les Histoires de vie. De l'invention de soi au projet de formation. Paris : Anthropos.

Delory-Momberger, C. (2005). Histoire de vie et recherche biographique en éducation. Paris : Anthropos.

Delory-Momberger, C. (2009). La condition biographique. Essais sur le récit de soi dans la modernité avancée. Paris : Téraèdre.

Dennett, D.C. (1993). La Conscience expliquée. Paris : Odile Jacob.

Dilthey, W. (1992 [1883]). Introduction aux sciences de l'esprit. Traduction et présentation de Sylvie Mesure. Paris : Cerf.

Dilthey, W. (1988 [1910]). L'édification du monde historique dans les sciences de l'esprit. Traduction, présentation et notes par Sylvie Mesure. Paris : Cerf.

Dufour, D.-R. (2011). L'individu qui vient... après le libéralisme. Paris : Denoël.

Foucault, M. (2001). Le sujet et le pouvoir. In Dits et écrits II. 1976-1988 (pp.10411062). Paris : Quarto Gallimard.

Franssen, A. (2006). L'État social actif et la nouvelle fabrique du sujet. In I. Astier \& N. Duvoux (dir.). La société biographique. Une injonction à vivre dignement (pp. 75-104). Paris : L'Harmattan.

Gazzaniga, M. S. (1994). Nature's Mind. Harmondsworth: Pinguin Books.

Gazzaniga, M. S. (2008). Human. The Science behind what makes us unique. Harper Collins.

Giddens, A. (1994). Les conséquences de la modernité. Paris : L’Harmattan.

Janet, P. (1935). Les Débuts de l'intelligence. Paris : Flammarion.

Janet, P. (1936). L'Intelligence avant le langage. Paris : Flammarion.

Lyotard, J.-F. (1979). La Condition postmoderne. Paris : Minuit.

Ricoeur, P. (1983). Temps et récit I. Paris : Seuil.

Ricoeur, P. (2005). Devenir capable, être reconnu. Esprit, 7, juillet 2005. 
Schapp, W. 1992 [1953]). Empêtrés dans des histoires. L'être de l'homme et de la chose. Traduction de J. Greisch. Paris : Cerf.

Victorri, B. (2002). Homo narrans. Le rôle de la narration dans l'émergence du langage. Langages, 146, 112-125.

Wieviorka, M. (2008). Neuf leçons de sociologie. Paris : Robert Laffont. 